\title{
Leaving the innermost stable circular orbit: the inner edge of a black-hole accretion disk at various luminosities
}

\author{
M. A. Abramowicz ${ }^{1,2,5,7}$, M. Jaroszyński ${ }^{3}$, S. Kato ${ }^{4}$, J.-P. Lasota ${ }^{5,6}$, A. Różańska ${ }^{2}$, and A. Sądowski ${ }^{2}$ \\ 1 Department of Physics, Göteborg University, 412-96 Göteborg, Sweden \\ e-mail: Marek.Abramowicz@physics.gu.se \\ 2 N. Copernicus Astronomical Center, Polish Academy of Sciences, Bartycka 18, 00-716 Warszawa, Poland \\ e-mail: [as; agata]@camk.edu.pl \\ 3 Warsaw University Observatory, Al. Ujazdowskie 4, 00-478 Warszawa, Poland \\ e-mail: mj@astrouw.edu.pl \\ 4 2-2-2 Shikanodai-Nishi, Ikoma-shi, Nara 630-0114, Japan \\ e-mail: kato.shoji@gmail.com, kato@kusastro.kyoto-u.ac.jp \\ 5 Institut d'Astrophysique de Paris, UMR 7095 CNRS, UPMC Univ Paris 06, 98bis Bd Arago, 75014 Paris, France \\ e-mail: lasota@iap.fr \\ 6 Jagiellonian University Observatory, ul. Orla 171, 30-244 Kraków, Poland \\ 7 Institute of Physics, Faculty of Philosophy and Science, Silesian University in Opava, Bezručovo nám. 13, 746-01 Opava, \\ Czech Republic
}

Received 19 March 2010 / Accepted 26 May 2010

\section{ABSTRACT}

\begin{abstract}
The "radiation inner edge" of an accretion disk is defined as the inner boundary of the region from which most of the luminosity emerges. Similarly, the "reflection edge" is the smallest radius capable of producing a significant X-ray reflection of the fluorescent iron line. For black hole accretion disks with very sub-Eddington luminosities these and all other "inner edges" coexist at the innermost stable circular orbit (ISCO). Thus, in this case, one may rightly consider ISCO as the unique inner edge of the black hole accretion disk. However, even at moderate luminosities, there is no such unique inner edge because differently defined edges are located at different places. Several of them are significantly closer to the black hole than ISCO. These differences grow with the increasing luminosity. For nearly Eddington luminosities, they are so huge that the notion of the inner edge loses all practical significance.
\end{abstract}

Key words. black hole physics - accretion, accretion disks

\section{Introduction}

Accretion flows onto black holes must change character before matter crosses the event horizon, for two reasons. First, matter must cross the black-hole surface at the speed of light as measured by a local inertial observer (see e.g., Gourgoulhon \& Jaramillo 2006), so that if the flow is subsonic far away from the black-hole (in practice it is always the case) it will have to cross the sound barrier (well) before reaching the horizon. This is the property of all realistic flows independent of their angular momentum. This sonic surface can be considered as the inner edge of the accretion flow.

The second reason is related to angular momentum. Far from the hole many (most probably most) rotating accretion flows adapt the Keplerian angular momentum profile. Because of the existence of the innermost stable circular orbit (ISCO), these flows must stop to be Keplerian there. At high accretion rates when pressure gradients become important, the flow may extend below the ISCO but the presence of the innermost bound circular orbit (IBCO) defines another limit to a circular flow (the absolute limit being given by the circular photon orbit, the CPO). These critical circular orbits provide another possible definition of the inner edge of the flow, in this case of an accretion disk.

The question is: what is the relation between the accretion flow edges? In the case of geometrically thin disks the sonic and
Keplerian edges coincide and one can define the ISCO as the inner edge of these disks. Paczyński (2000) showed rigorously that independent of e.g., viscosity mechanism and the presence of magnetic fields, the ISCO is the universal inner disk's edge for not too-high viscosities. The case of thin disks is therefore settled ${ }^{1}$.

However, this is not the case for non-thin accretion disks, i.e., of medium and high luminosities. The problem of defining the inner edge of an accretion disk is not just a formal exercise. Afshordi \& Paczyński (2003) explored several reasons which made discussing the precise location of inner edge $r=r_{\text {in }}$ of the black hole accretion disks an interesting and important issue. One of them was,

Theory of accretion disks is several decades old. With time ever more sophisticated and more diverse models of accretion onto black holes have been introduced. However, when it comes to modeling disk spectra, conventional steady state, geometrically thin-disk models are still used, adopting

1 Penna et al. (2010) studied the effects of magnetic fields on thin accretion disk (the disk thickness $H / r \lesssim 0.07$, which corresponds to $L \lesssim 0.2 L_{\text {Edd }}$ ). They found that to within a few percent the magnetized disks are consistent with the Novikov \& Thorne (1973) model, in which the inner edge coincides with the ISCO. 
the classical "no torque" inner boundary condition at the marginally stable orbit.

The clearest illustration of this is the state-of-art work on measuring the black hole spin $a$ in the microquasar GRS 1915+105 by fitting its observed "thermal state" spectra to those calculated (e.g. McClintock et al. 2006; Middleton et al. 2009). These works use a general relativistic version of the classical ShakuraSunyaev thin accretion disk model developed by Novikov \& Thorne (1973). The Novikov-Thorne model assumes that the inner edge of the disk $r_{\text {in }} \equiv r_{\text {ISCO }}$ is also the innermost boundary of the radiating region.

Because the black hole mass of GRS $1915+105$ is known and therefore fixed $\left(M_{0}=14 M_{\odot} \pm 4 M_{\odot}\right)$, the surface area $A$ of the radiating region, calculated in the model, depends only on the black hole unknown spin, $a^{*}\left(a^{*}=J c / G M^{2}\right.$ with $J$ being the total angular momentum of the black hole). In the thermal state, the disk spectrum is close to that of a sum of black body contributions from different radial locations. Its shape is determined by the radial distribution of temperature, which in the NovikovThorne model depends on the spin, $T=T\left(r, a^{*}\right)$. The total radiation power $L$ is determined by the "averaged" temperature $T_{0}=T_{0}\left(a^{*}\right)$ and the surface area $A=A\left(a^{*}\right)$ of the radiating region, $L=\sigma T_{0}^{4} A$. By calculating the spectral shape and power for different $a^{*}$ in the Novikov-Thorne model, one may find the bestfit estimates of the spin-dependent temperature and area. This is just the main idea of the spin estimate; details of the fitting are far more complex (see McClintock et al. 2006; Davis et al. 2005, Staub et al., in prep.) and include, for example, a heuristic way of treating a contribution of scattering in accretion disk atmosphere (i.e., the "hardening factor"). Results obtained in this way by McClintock et al. (2006) for GRS 1915+105 showed that $a^{*}=0.99$ for the luminosity range $L<0.2 L_{\text {Edd }}$. However, for $L>0.2 L_{\mathrm{Edd}}$, the spin estimated by McClintock et al. (2006) was much lower, $a^{*} \approx 0.8$. The inconsistent spin estimates at different luminosities may indicate that some assumptions adopted by the Novikov-Thorne model are wrong at high luminosities.

This is not a surprise, because there are several physical effects known to be important at high luminosities, that are ignored in the classical Shakura-Sunyaev and Novikov-Thorne thin accretion disk models. These effects are properly included in the slim $^{2}$ accretion disks models, introduced by Abramowicz et al. (1988). Advection is perhaps the most well-known of these "slim disk effects", but in the present context the significant stress caused by the radial pressure gradient (for thin disks $\mathrm{d} P / \mathrm{d} r \approx 0$ ) is equally important. The stress firmly retains matter well inside ISCO and as a result of this, at high luminosities the edge of the plunge-in region may be considerably closer to the black hole than the $\mathrm{ISCO}^{3}$.

Slim disks are assumed to be stationary and axially symmetric. They are described by vertically integrated NavierStokes hydrodynamical equations; no magnetic fields are considered. The effective viscosity, assumed to be generated by the MHD turbulence (Balbus \& Hawley 1991), is described by the " $\alpha P$ " Shakura-Sunyaev ansatz. We neglect additional effects connected to turbulent components of magnetic fields, winds, or disk corona, basing our study on conservation laws alone.

\footnotetext{
2 The names thin and slim refer to the dimensionless vertical geometrical thickness, $h=H / r$. For thin disks, it must be $h \ll 1$, while for slim disks a weaker condition $h<1$ holds.

${ }^{3}$ Matter may also be retained well inside the ISCO by magnetic stresses, as pointed out by many authors; see e.g. a semi-analytic model by Narayan et al. (2003), or MHD numerical simulations by Noble et al. (2010), and references quoted in these papers.
}

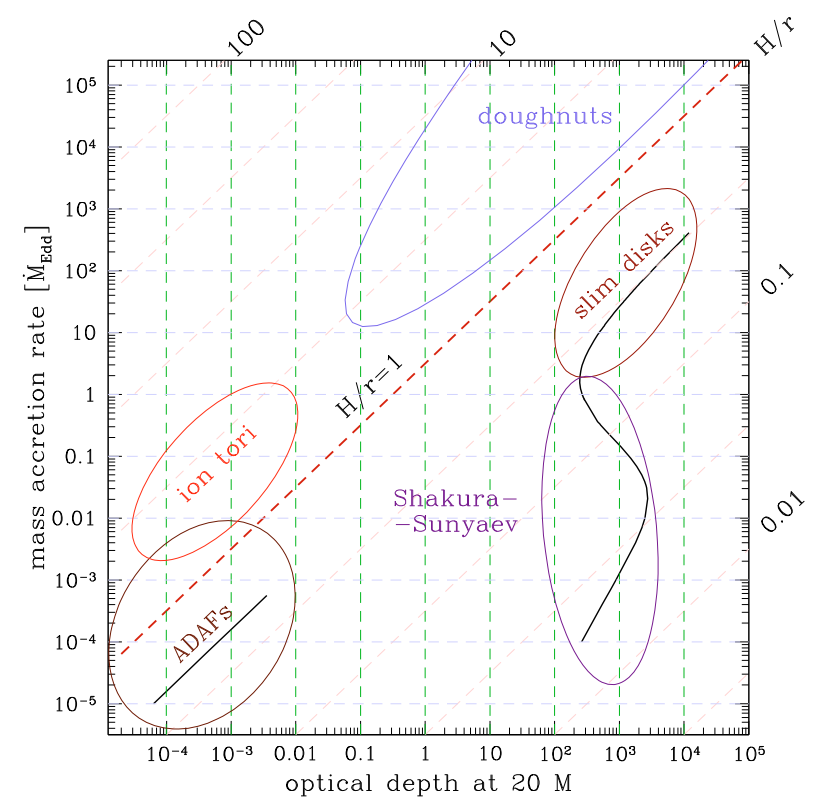

Fig. 1. This figure illustrates a few of the most well-known analytic and semi-analytic solutions of the stationary black hole accretion disks. Their location in the parameter space approximately corresponds to viscosity $\alpha=0.1$ and radius $r=20 \mathrm{M}$.

Figure 1 shows the slim disk location with respect to other analytic and semi-analytic disk models ${ }^{4}$, in the parameter space $[\tau, h, \dot{m}]$ described by the vertical optical depth $\tau$, dimensionless vertical thickness $h=H / r$, and dimensionless accretion rate $\dot{m}=\dot{M} / \dot{M}_{\text {Edd }}$, where $\dot{M}_{\text {Edd }}=16 L_{\text {Edd }} / c^{2}$ is the critical accretion rate approximately corresponding to the Eddington luminosity $\left(L_{\mathrm{Edd}}=10^{38} \mathrm{M} / \mathrm{M}_{\odot} \mathrm{erg} \mathrm{s}^{-1}\right)$ in case of a disk around a non-rotating black hole ${ }^{5}$.

In this paper, we discuss for the first time in detail the properties of the inner edge of the slim accretion disks around rotating black holes, using models similar to those calculated by Sadowski $(2009)^{6}$. For convenience, we briefly recall the slim disk basic equations in Appendix A. In Sect. 2, we list six possible definitions of the inner edge. These definitions reflect different (but partially overlapping) physical meanings and different practical astrophysical applications. In the subsequent Sects. 3-8, we calculate the slim disk locations of these six inner edges, and discuss their astrophysical relevances. Some of the results presented here were found previously both by ourselves and other authors in a different context of Polish doughnuts (i.e. thick accretion disks; see e.g. the short review by Paczyński 1998); we refer also to Paczyński (2000) and Afshordi \& Paczyński (2003).

\section{Definitions of the inner edge}

Krolik \& Hawley (2002) proposed several "empirical" definitions of the inner edge, each serving a different practical purpose (see also the follow-up investigation by Beckwith et al. 2002).

\footnotetext{
4 For detailed reviews of these solutions see, http://www.scholarpedia.org/article/Accretion_discs or Kato et al. (2008).

5 Two points about notation: (i) many authors use a different definition, $\dot{M}_{\text {Edd }}=L_{\text {Edd }} / c^{2}$. (ii) In this paper, we often use the $c=1=G$ convention in which $M=r_{G}=G M / c^{2}$.

6 At http://users.camk.edu.pl/as/slimdisks we provide a very detailed database for these solutions, which covers the whole parameter space relevant to microquasars and AGN.
} 

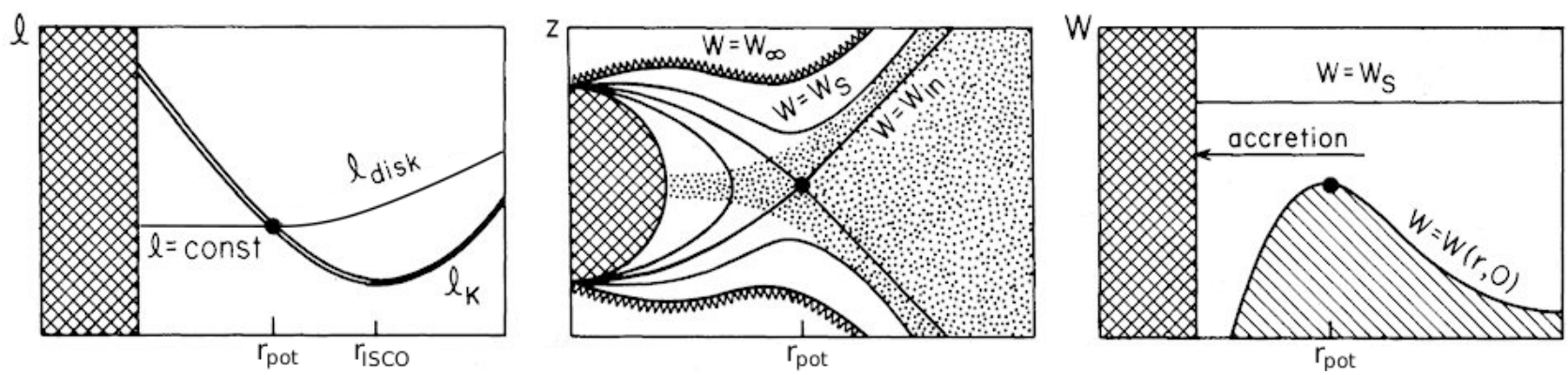

Fig. 2. An illustrative visualisation of the Roche lobe overflow. The leftmost panel schematically presents disk angular momentum profile and its relation to the Keplerian distribution. The middle panel shows the equipotential surfaces. The dotted region denotes the volume filled with accreting fluid. The rightmost panel presents the potential barrier at the equatorial plane $(z=0)$ and the potential of the fluid $\left(W_{S}\right)$ overflowing the barrier. The figure is taken from http://www.scholarpedia.org/article/Accretion_discs.

We add to these a few more definitions. The list of the inner edges considered in this paper consists of ${ }^{7}$,

[1] The potential spout edge $r_{\text {in }}=r_{\text {pot }}$, where the effective potential forms a self-crossing Roche lobe, and accretion is governed by the Roche lobe overflow.

[2] The sonic edge $r_{\text {in }}=r_{\text {son }}$, where the transition from subsonic to transonic accretion occurs. Hydrodynamical disturbances do not propagate upstream a supersonic flow, and therefore the subsonic part of the flow is "causally" disconnected from the supersonic part.

[3] The variability edge $r_{\text {in }}=r_{\mathrm{var}}$, the smallest radius where orbital motion of coherent spots may produce quasi-periodic variability.

[4] The stress edge $r_{\text {in }}=r_{\text {str }}$, the outermost radius where the Reynolds stress is small, and plunging matter has no dynamical contact with the outer accretion flow.

[5] The radiation edge $r_{\text {in }}=r_{\text {rad }}$, the innermost place from which significant luminosity emerges.

[6] The reflection edge $r_{\text {in }}=r_{\text {ref }}$, the smallest radius capable of producing significant fluorescent iron line.

In the next six sections, we discuss these six edges one by one.

\section{The potential spout edge}

The idea of the "relativistic Roche lobe overflow" governing accretion close to the black hole was first explained by Paczyński (see Kozłowski et al. 1978). It was later explored in detail by many authors analytically (e.g. Abramowicz 1981, 1985) and by large-scale hydrodynamical simulations (e.g. Igumenshchev \& Beloborodov 1997). It became a standard concept in black hole accretion theory. Figure 2 schematically illustrates the Roche lobe overflow mechanism. The leftmost panel presents a demonstrative profile of disk angular momentum, which reaches the Keplerian value at the radius corresponding to the self-crossing of the equipotential surfaces presented in the middle panel. To flow through this "cusp", matter must have potential energy higher than the value of the potential at this point, i.e., the "potential barrier" is crossed only when the matter overflows its Roche lobe. Precise profiles of the potential barriers and the angular momentum, calculated with the slim disk model, are presented in Figs. 3 and 4, respectively.

\footnotetext{
7 Krolik \& Hawley defined the inner edges [4], [5] and [6] and in addition a seventh edge [7], the turbulence edge, where flux-freezing becomes more important than turbulence in determining the magnetic field structure. Magnetic fields are not considered for slim accretion disks, and we therefore do not discuss [7].
}

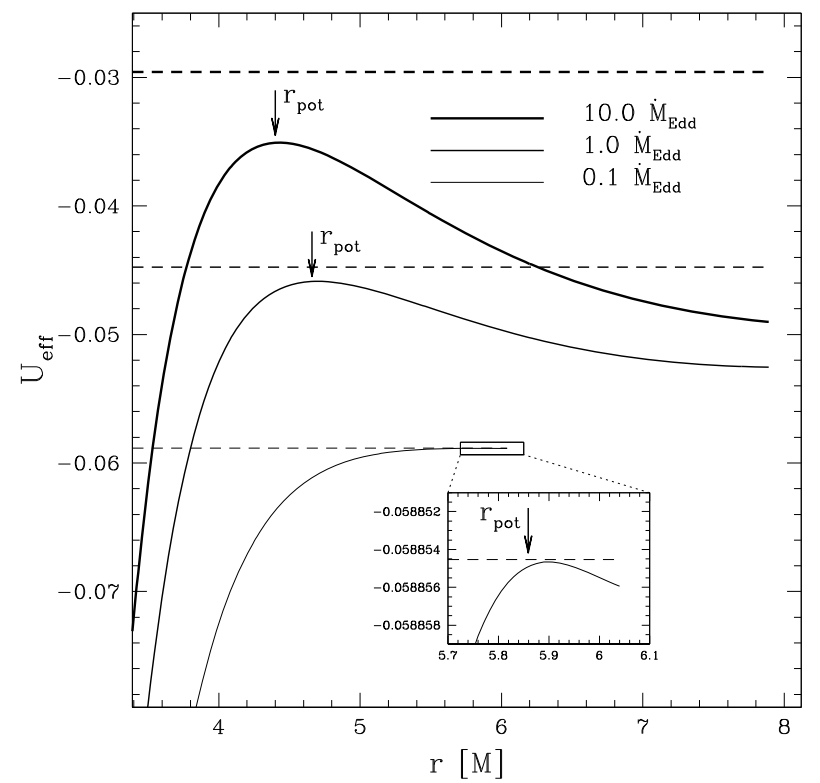

Fig. 3. Profiles of the effective potential near the potential barrier (solid lines) for different accretion rates, $\alpha=0.01$ and $a^{*}=0$. The arrows indicate locations of the inner edge potential spout $-r_{\text {pot }}$ corresponding to the top of the potential barrier. The horizontal dashed lines present the energy of the gas overflowing the barrier calculated at $r_{\text {pot }}$.

The potential difference between the horizon and the spout is infinite, and therefore no external force can prevent the matter located there from plunging into the black hole. At radii greater that $r_{\text {pot }}$, the potential barrier at $r=r_{\text {pot }}$ retains the matter inside this radius. We note, that because the dynamical equilibrium is given (approximately) by $\nabla_{i} \mathcal{U}_{\text {eff }}=\nabla_{i} P / \rho$, with $\rho$ being the density, one may also say that it is the pressure gradient (the pressure stress) that holds the matter within $r_{\text {pot }}$.

The specific angular momentum in the Novikov-Thorne model is assumed to be Keplerian. Slim disk models do not a priori assume an angular momentum distribution, but selfconsistently calculate it from the relevant equations of hydrodynamics Eqs. (A.1)-(A.8). These calculations indicate that the type of angular momentum distribution depends on whether the accretion rate and viscosity constrain the flow to be either disklike or Bondi-like type.

In the Bondi-type accretion flows, the angular momentum is everywhere sub-Keplerian, $\mathcal{L}<\mathcal{L}_{\mathrm{K}}$. These flows are typical of high viscosities and high accretion rates, as the case of $\alpha=0.1$ and $\dot{m}=10$ shown in Fig. 4. This is the only Bondi-like flow in 


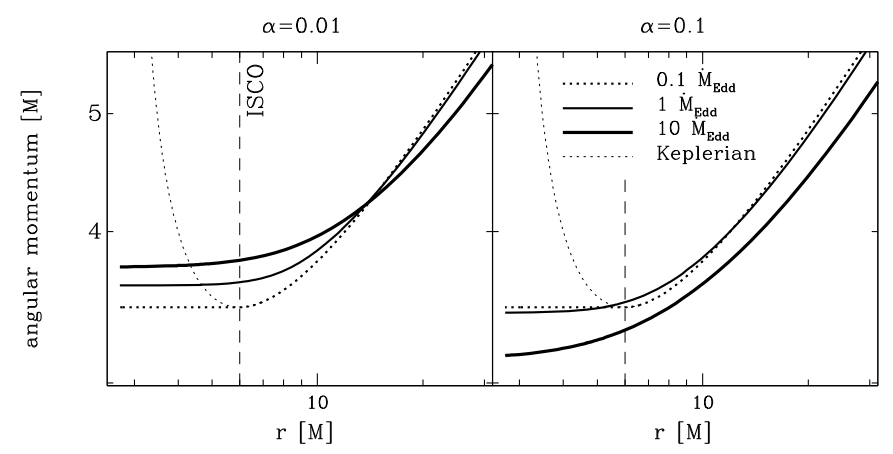

Fig. 4. Angular momentum profiles for slim disk solutions with $\alpha=0.01$ (left panel) and $\alpha=0.1$ (right panel). In both panels, three curves are presented for sub-Eddingtonian, Eddingtonian, and super-Eddingtonian accretion rates. The thin dotted line presents the Keplerian angular momentum profile.

this figure. In the disk-like accretion flows, the angular momentum of the matter in the disk is sub-Keplerian everywhere, except the strong-gravity region $r_{\text {pot }}<r<r_{\text {cen }}$, where the flow is superKeplerian, $\mathcal{L}>\mathcal{L}_{\mathrm{K}}$. The radius $r_{\text {cen }}>r_{\text {ISCO }}$ corresponds to the ring of the maximal pressure in the accretion disk. This is also the minimum of the effective potential. The radius $r_{\text {pot }}<r_{\text {ISCO }}$ delineates a saddle point for both pressure and effective potential; this is also the location of the "potential spout inner edge", $r_{\text {in }}=r_{\text {pot }}$.

We note that in the classic solutions for spherically accretion flows found by Bondi (1952) the viscosity is unimportant and the sonic point is saddle, while in the "Bondi-like" flows discussed here, angular momentum transport by viscosity is essentially important and the sonic point is usually nodal. Therefore, one should keep in mind that the difference between these types of accretion flows is also determined by the relative importance of pressure and viscosity. For this reason, a different terminology is often used. Instead of "disk-like", one uses the term "pressuredriven", and instead of "Bondi-like", one uses "viscosity-driven" (see e.g. Matsumoto et al. 1984; Kato et al. 2008).

From the above discussion, it is clear that the location of this particular inner edge $r_{\text {pot }}$ is formally given as the smaller of the two roots, $r_{ \pm}=\left(r_{+}, r_{-}\right)$, of the equation

$\left[\mathcal{L}(r)-\mathcal{L}_{\mathrm{K}}(r)\right]_{r=r_{ \pm}}=0$.

The larger root corresponds to $r_{\text {cen }}$. Obviously, Eq. (1) has always a solution for the disk-like flows, and never for the Bondilike flows. Figure 5 shows a division of the parameter space into regions occupied by Bondi-like and disk-like flows.

The location of the potential spout inner edge $r_{\text {pot }}$ is shown in Fig. 6 for $\alpha=0.01$. We note that for low accretion rates, $\dot{m} \lesssim 0.3$, the location of the potential spout inner edge coincides with ISCO. At $\dot{m} \approx 0.3$, the location of the potential spout jumps to a new position, which is close to the radius of the innermost bound circular orbit, $r_{\mathrm{IBCO}}$. This behavior has long been recognized first by Kozłowski et al. (1978) for Polish doughnuts, and then by Abramowicz et al. (1988) for slim disks. We conclude the section on the potential spout inner edge by giving an approximate formula for its location

$$
\begin{aligned}
r_{\text {pot }}\left(a^{*}, \dot{m}\right)= & \operatorname{Min}\left[\left(0.275-0.410 a^{*}+0.143 a^{* 2}\right) \dot{m}^{-1.4}\right. \\
& \left.+4.45-4.87 a^{*}+8.06 a^{* 2}-6.38 a^{* 3} ; 0.985 r_{\text {ISCO }}\right] .
\end{aligned}
$$

The formula in Eq. (2) is valid for $\alpha=0.01$.

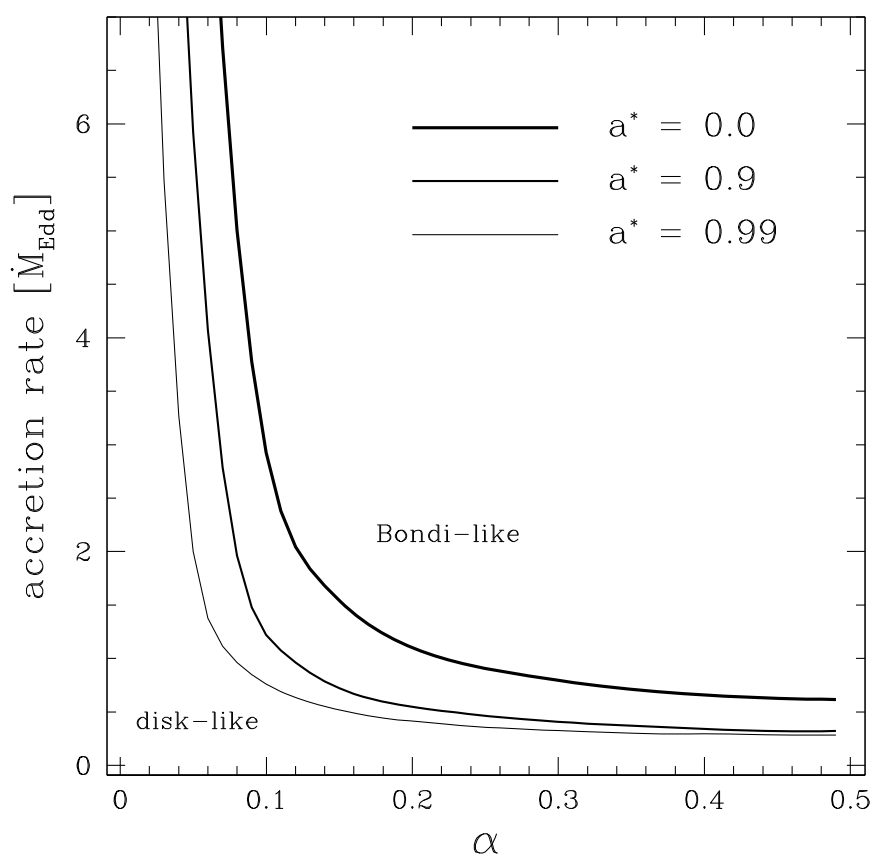

Fig. 5. Location of the Bondi-like and the disk-like slim accretion disks in the $[\alpha, \dot{m}]$ parameter space. The Bondi-like accretion flows are everywhere sub-Keplerian. Disk-like flows are sub-Keplerian for most radii, but also have a super-Keplerian part around ISCO.

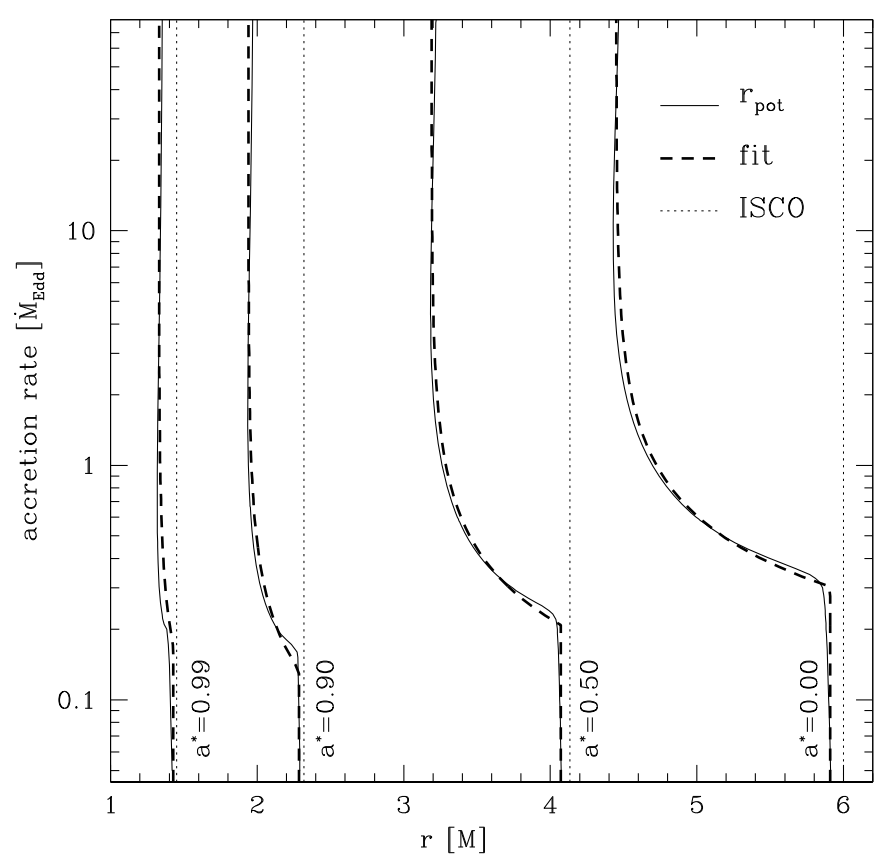

Fig. 6. Location of the potential spout inner edge $r_{\text {pot }}$ for viscosity $\alpha=$ 0.01 and $a^{*}=0$. Solid lines show the exact location of $r_{\text {pot }}$ given by Eq. (1). The approximation given by Eq. (2) is shown by dashed lines, and the location of ISCO by dotted lines.

\section{The sonic edge}

By a series of algebraic manipulations, one reduces the slim disk equations in Eqs. (A.1)-(A.8) to a set of two ordinary differential equations for two dependent variables, e.g. the Mach number $\eta=-V / c_{S}^{2}=-V \Sigma / P$ and the angular momentum $\mathcal{L}=-u_{\phi}$,

$\frac{\mathrm{d} \ln \eta}{\mathrm{d} \ln r}=\frac{\mathcal{N}_{1}(r, \eta, \mathcal{L})}{\mathcal{D}(r, \eta, \mathcal{L})} \quad \frac{\mathrm{d} \ln \mathcal{L}}{\mathrm{d} \ln r}=\frac{\mathcal{N}_{2}(r, \eta, \mathcal{L})}{\mathcal{D}(r, \eta, \mathcal{L})}$ 


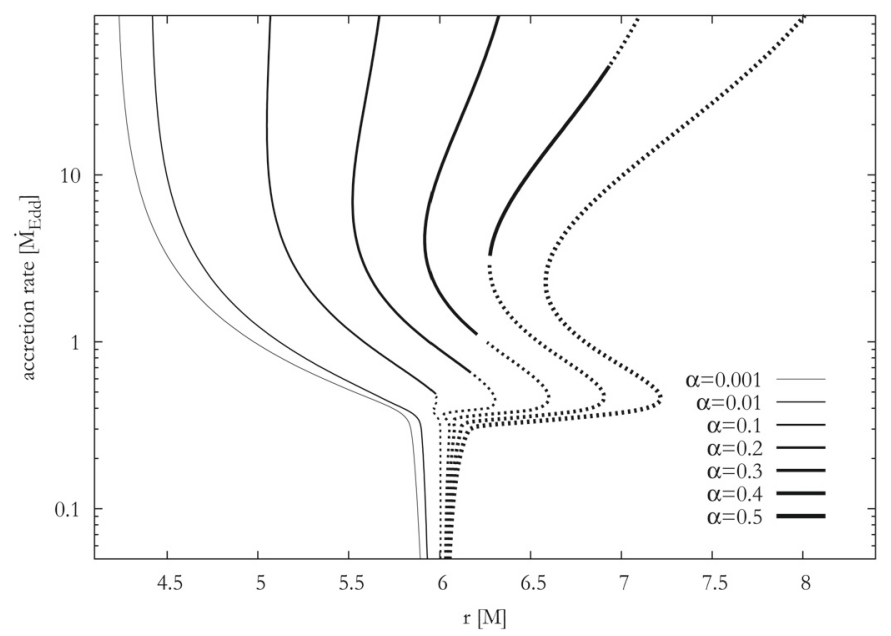

Fig. 7. Location of the sonic point as a function of the accretion rate for different values of $\alpha$, for a non-rotating black hole, $a^{*}=0$. The solid curves are for saddle-type solutions, while the dotted curves present nodal-type regimes.

For a non-singular physical solution, the nominators $\mathcal{N}_{1}$ and $\mathcal{N}_{2}$ must vanish at the same radius as the denominator $\mathcal{D}$. The denominator vanishes at the sonic edge (or sonic radius) where the Mach number is close to unity, i.e.

$\mathcal{D}(r, \eta, \mathcal{L})_{\mid r=r_{\text {son }}}=0$.

For low mass accretion rates, lower than about $0.3 \dot{M}_{\text {Edd }}$ in the case of $a^{*}=0$, the sonic edge $r_{\text {son }}$ is close to ISCO, independently of the viscosity $\alpha$, as Fig. 7 shows. At about $0.3 \dot{M}_{\text {Edd }}$, a qualitative change occurs, resembling a "phase transition" from the Shakura-Sunyaev behavior to a very different slim-disk behavior.

For higher accretion rates, the location of the sonic point significantly departs from ISCO. For low values of $\alpha$, the sonic point moves closer to the horizon down to $\sim 4 \mathrm{M}$ for $\alpha=0.001$. For $\alpha>0.2$, the sonic point moves outward with increasing accretion rate reaching values as high as $8 \mathrm{M}$ for $\alpha=0.5$ and $100 \dot{M}_{\text {Edd }}$. This effect was first noticed for low accretion rates by Muchotrzeb-Czerny (1986) and later investigated for a wide range of accretion rates by Abramowicz et al. (1988), who explained it in terms of the disk-Bondi dichotomy. The dependence of the sonic point location on the accretion rate in the nearEddington regime is more complicated and is related to, for this range of accretion rates, the transition from the radiatively efficient disk to the slim disk occuring close to the sonic radius.

The topology of the sonic point is important, because physically acceptable solutions must be of the saddle or nodal type, the spiral type being forbidden. The topology may be classified by the eigenvalues $\lambda_{1}, \lambda_{2}, \lambda_{3}$ of the Jacobi matrix

$\mathcal{J}=\left[\begin{array}{lll}\frac{\partial \mathcal{D}}{\partial r} & \frac{\partial \mathcal{D}}{\partial \eta} & \frac{\partial \mathcal{D}}{\partial \mathcal{L}} \\ \frac{\partial \mathcal{N}_{1}}{\partial r} & \frac{\partial \mathcal{N}_{1}}{\partial \eta} & \frac{\partial \mathcal{N}_{1}}{\partial \mathcal{L}} \\ \frac{\partial \mathcal{N}_{2}}{\partial r} & \frac{\partial \mathcal{N}_{2}}{\partial \eta} & \frac{\partial \mathcal{N}_{2}}{\partial \mathcal{L}}\end{array}\right]$

Because $\operatorname{det}(\mathcal{J})=0$, only two eigenvalues $\lambda_{1}, \lambda_{2}$ are non-zero, and the quadratic characteristic equation that determines them takes the form,

$2 \lambda^{2}-2 \lambda \operatorname{tr}(\mathcal{J})-\left[\operatorname{tr}\left(\mathcal{J}^{2}\right)-\operatorname{tr}^{2}(\mathcal{J})\right]=0$

The nodal type is given by $\lambda_{1} \lambda_{2}>0$ and the saddle type by $\lambda_{1} \lambda_{2}<0$, as indicated in Fig. 7 by the dotted and the solid

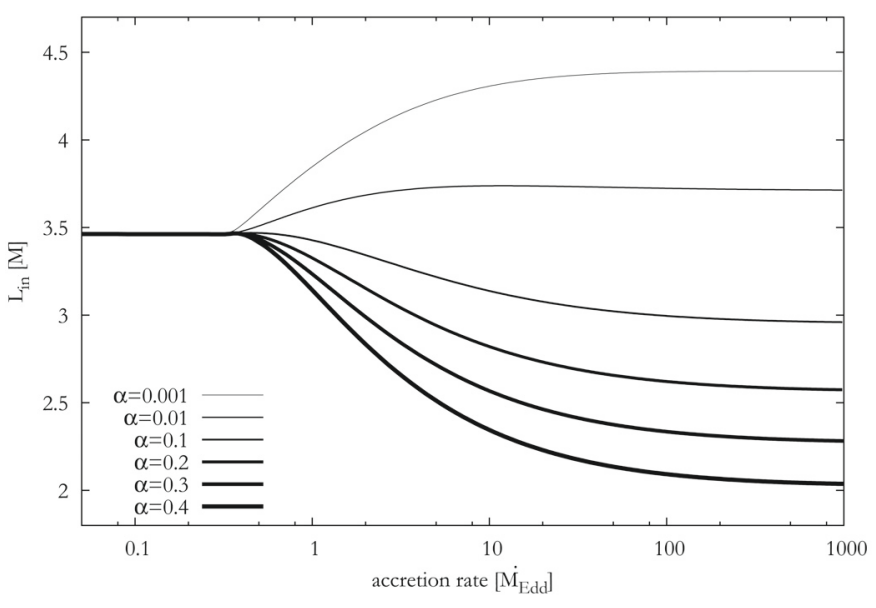

Fig. 8. Dependence of the angular momentum at the horizon on accretion rate for solutions with different values of $\alpha$ for $a^{*}=0$.

lines, respectively. For the lowest values of $\alpha$, only the saddletype solutions exist. For moderate values of $\alpha(0.1 \leq \alpha \leq 0.4)$, the topological type of the sonic point changes at least once with increasing accretion rate. For the highest $\alpha$, solutions have only nodal-type critical points.

The extra regularity conditions at the sonic point $\mathcal{N}_{i}(r, \eta, \mathcal{L})=0$ are satisfied only for one particular value of the angular momentum at the horizon, which is the eigenvalue of the problem. The parameter $\mathcal{L}_{\text {in }}$ is not known a priori, and should be found. Figure 8 shows how $\mathcal{L}_{\text {in }}$ depends on both the accretion rate and the $\alpha$ viscosity parameter.

\section{The variability edge}

Axially symmetric and stationary states of slim accretion disks are, obviously, theoretical idealizations. Real disks are non-axial and non-steady. In particular, one expects transient coherent features at accretion disk surfaces - clumps, flares, and vortices. The orbital motion of these features could quasi-periodically modulate the observed flux of radiation, mostly by means of the Doppler effect and the relativistic beaming. We define $\Pi$ to be the "averaged" variability period, and $\Delta \Pi$ a change in the period during one period caused by the radial motion of a spot. The variability quality factor $Q$ may be estimated by,

$\frac{1}{Q}=\frac{\Delta \Pi}{\Pi}=\frac{\Delta \Omega}{\Omega}=\frac{1}{\Omega} \frac{\mathrm{d} \Omega}{\mathrm{d} r} \Delta r=2 \pi \frac{1}{\Omega^{2}} \frac{\mathrm{d} \Omega}{\mathrm{d} r} \frac{u^{r}}{u^{t}}$,

where $u^{r} / u^{t}=\mathrm{d} r / \mathrm{d} t$ and $u^{r}$ and $u^{t}$ are contravariant components of the four velocity. The period is given in terms of the orbital angular velocity by $\Pi=2 \pi / \Omega$. Using the relations (see Appendix A for the explanation of the notation used)

$u^{r}=\frac{V}{\sqrt{1-V^{2}}} \frac{\sqrt{\Delta}}{r}$
$u^{t}=\frac{\gamma \sqrt{A}}{r \sqrt{\Delta}}=\frac{\sqrt{A}}{r \sqrt{\Delta}} \frac{1}{\sqrt{\left(1-V^{2}\right)\left(1-\left(\tilde{V}^{\phi}\right)^{2}\right)}}$

where $V$ is the radial velocity measured by an observer corotating with the fluid, one obtains

$Q=\frac{1}{2 \pi}\left|\frac{\mathrm{d} \log \Omega}{\mathrm{d} \log r}\right|^{-1}\left|\frac{\bar{V}^{\phi}}{V}\right| f^{*}\left(a^{*}, r\right)$, 


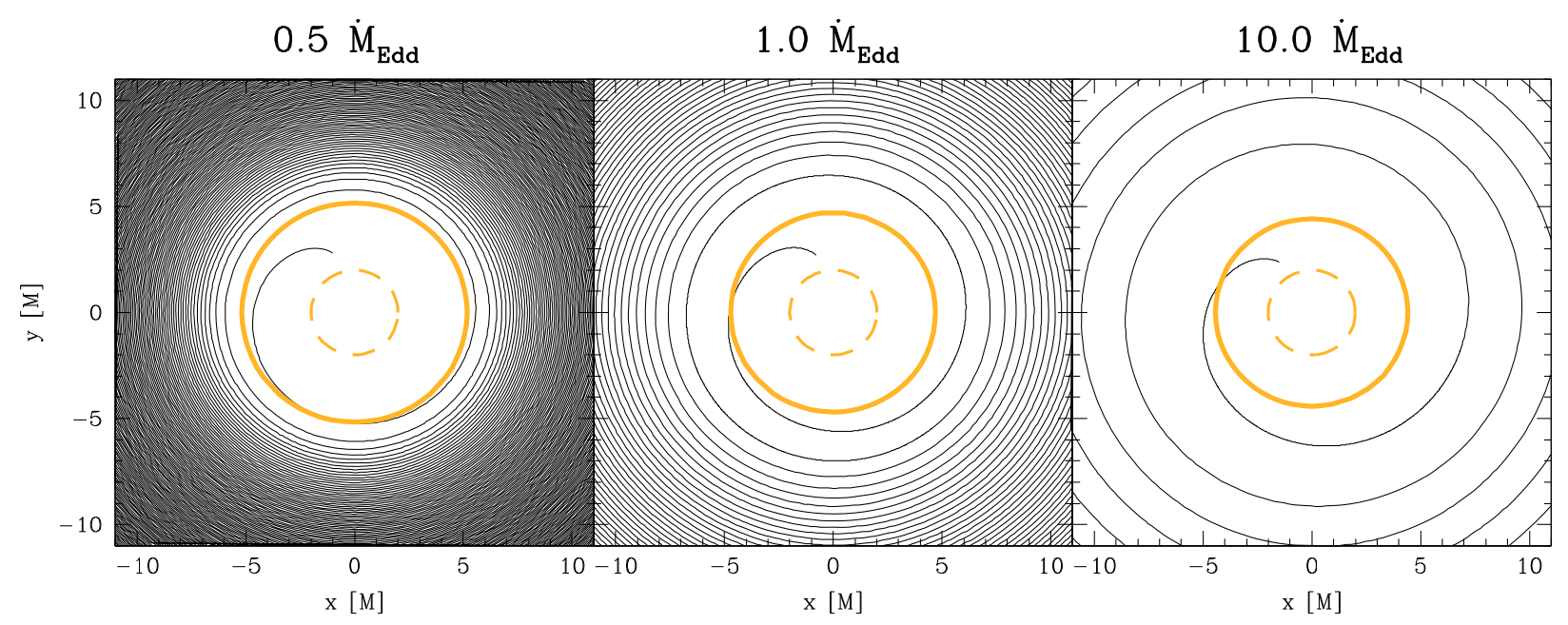

Fig. 9. The fluid flow trajectories in slim accretion disks shown by thin solid lines for different accretion rates. Locations of $r_{\text {pot }}$ and the location of black hole horizon are shown by thick gray solid and broken lines, respectively. For low accretion rates, the pattern of trajectories consists of very tight spirals (almost circles) for $r>r_{\mathrm{pot}} \approx r_{\mathrm{ISCO}}$ and very wide spirals (almost a radial fall) for $r<r_{\mathrm{pot}}$. In this case, there is a sharp transition from almost circular motion to almost radial free-fall that clearly defines the variability edge as $r_{\mathrm{var}}=r_{\mathrm{ISCO}}$. For higher accretion rates, the fluid trajectories are wide open spirals in the whole inner region of the flow and the variability edge makes no sense.

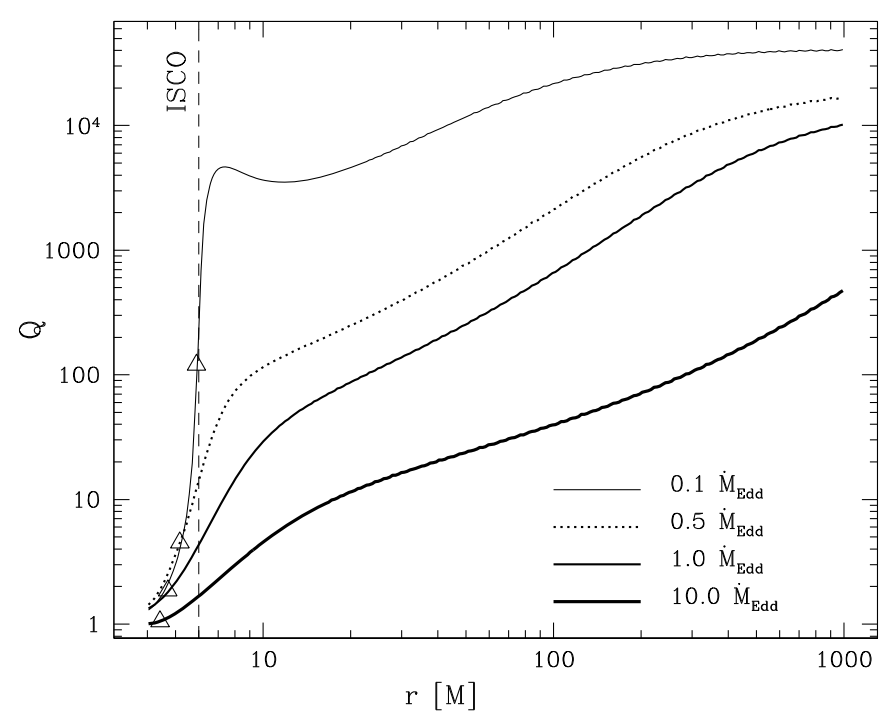

Fig. 10. The quality factor $Q$ profiles for different accretion rates. Triangles show $r_{\text {pot }}$ for each rate. The vertical dashed line denotes the location of ISCO.

where

$$
\begin{aligned}
& f^{*}\left(a^{*}, r\right) \equiv \frac{r^{3}}{\sqrt{\Delta A}}=+\left[1-X-X^{2} a^{* 2}\left(a^{* 2}+1\right)-X^{5} a^{* 4}\right]^{-1 / 2}, \\
& \bar{V}^{\phi}=\frac{V^{\phi}}{\sqrt{1-\left(V^{\phi}-\omega \tilde{R}\right)^{2}}}
\end{aligned}
$$

and $X=2 r_{G} / r$. From Eqs. (A.2) and (A.5), it is clear than $\Delta A>$ 0 outside the black hole horizon. We note that in the Newtonian limit it is $X \ll 1$ and one has $f^{*}\left(a^{*}, r\right)=1$. In this limit, $V$ and $\bar{V}^{\phi}$ are the radial and azimuthal components of velocity, and Eq. (9) takes its obvious Newtonian form.

The behavior of the quality factor $Q$ is shown in Fig. 10 . Profiles for four accretion rates are drawn. As Fig. 9 shows, the lower accretion rate the smaller radial velocity component, hence the quality factor $Q$ in general increases with decreasing accretion rate. For the lowest values of $\dot{m}$, a rapid drop is visible at ISCO corresponding to the change in the nature of the flow (gas enters the free-fall region below ISCO). For higher accretion rates, this behaviour is suppressed as the trajectories become wide open spirals well outside ISCO.

We note that our definition given in Eq. (7) of the quality factor $Q$, essentially agrees with a practical definition of the variability quality factor $Q_{0}$ defined by observers with the help of the observationally constructed Fourier variability power spectra, $I(v)$. Here $I(v)$ is the observed variability power (i.e. the square of the observed amplitude) at a particular observed variability frequency $v$. Any observed quasi periodic variability of the frequency $\sim v_{0}$ can clearly be seen in the power spectrum as a local peak in $I(v)$, centered on a particular frequency $v_{0}$. The half-width $\Delta v$ of the peak defines the variability quality factor by $Q_{0}=v / \Delta v_{0}$.

Quasi-periodic variability of $\mathrm{kHz}$ frequencies, called $\mathrm{kHz} \mathrm{QPO}$, is observed from several low-mass neutron star and black hole binaries. In a pioneering and important piece of research, Barret et al. (2005) carefully measured the quality factor for a particular source in this class (4U 1608-52) and found that $Q_{0} \sim 200$, i.e. that the $\mathrm{kHz}$ signals are very coherent. They argued that $Q_{0} \sim 200$ cannot be due to kinematic effects in the orbital motion of hot spots, clumps, or other similar features located at the accretion disk surface, because these features are too quickly sheared out by the differential rotation of the disk (see also Bath et al. 1974; Pringle 1981). They also argued that although coherent vortices may survive much longer times at the disk surface (e.g. Abramowicz et al. 1995), if they participate in the inward radial motion, the observed variability $Q_{0}$ cannot be high. Our results shown in Fig. 10 illustrate and strengthen this point. We also agree with the conclusion reached by Barret et al. (2005) that the observational evidence against orbiting clumps as a possible explanation of the phenomenon of $\mathrm{kHz} \mathrm{QPO}$, seems to indicate that this phenomenon is probably caused by the accretion disk global oscillations ${ }^{8}$. For excellent reviews of the QPO oscillatory models, we refer to Wagoner (1999) and Kato (2001).

\footnotetext{
8 Barret et al. (2005) also found how $Q_{0}$ varies in time for each of the two individual oscillations in the "twin-peak QPO". This places strong observational constraints on possible oscillatory models of the twin peak kHZ QPO; see also Boutelier et al. (2010).
} 
M. A. Abramowicz et al.: Leaving the ISCO
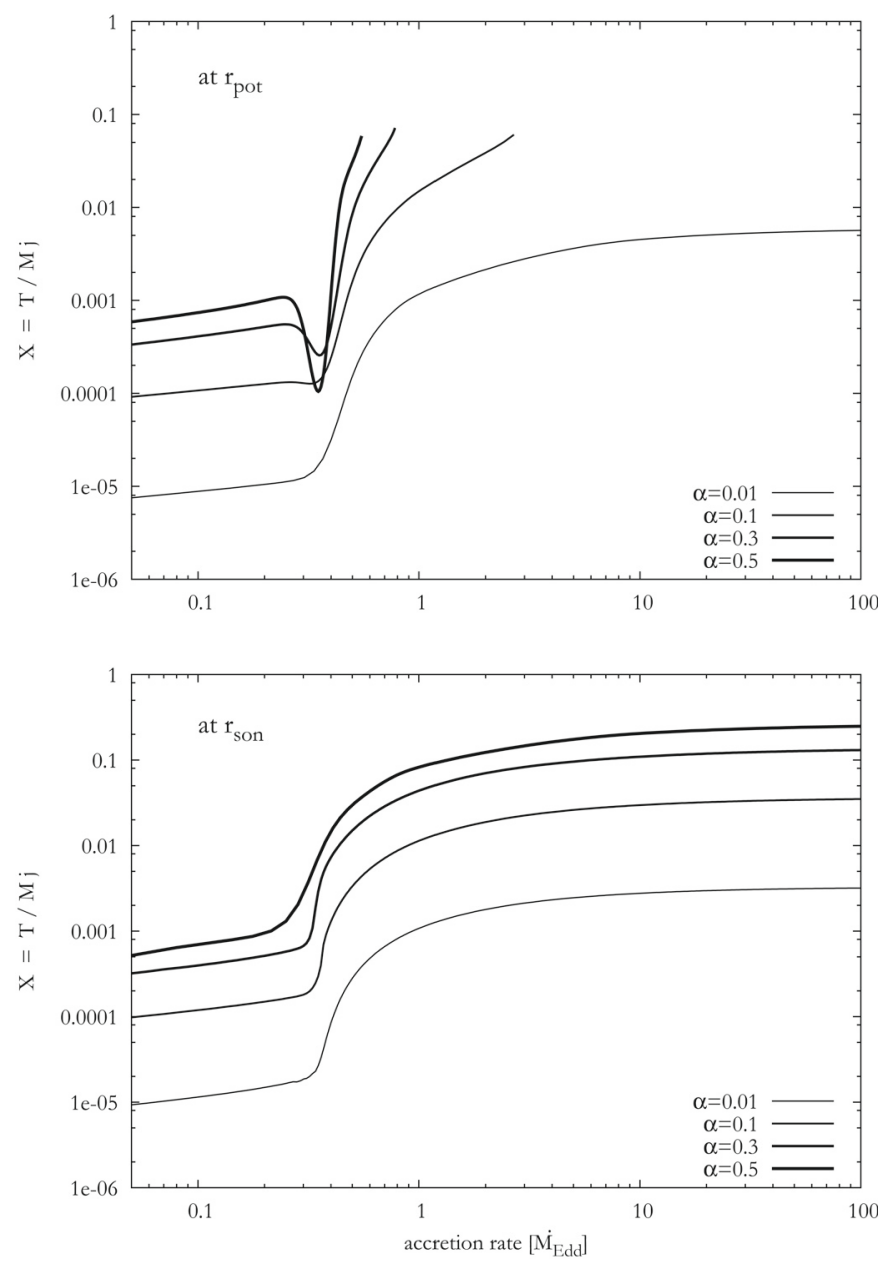

Fig. 11. Ratio of the angular momentum flux caused by torque to the flux caused by advection calculated at $r_{\text {pot }}$ (top) and $r_{\text {son }}$ (bottom panel) versus mass accretion rate for a number of values of $\alpha$ and $a^{*}=0$. The $r_{\text {pot }}$ profiles for high viscosities terminate when the disk enters the Bondi-like regime.

Although clumps, hot-spots, vortices or magnetic flares cannot explain the coherent $\mathrm{kHz}$ QPOs with $Q_{0} \sim 200$, they certainly are important in explaining the continuous, featureless Fourier variability power spectra (see e.g. Abramowicz et al. 1991; Schnittman 2005; Pecháček et al. 2008, and references quoted there). Our results shown in Fig. 10 indicate that: (i) at low accretion rates, a sharp high-frequency cut-off in $I(v)$ may be expected at about the ISCO frequency; (ii) at high accretion rates, there should be no cut-off in $I(v)$ at any frequency; and (iii) the logarithmic slope $p=(\mathrm{d} \ln I / \mathrm{d} \ln v)$ should depend on $\dot{m}$.

A more quantitative description of these points (i)-(iii) will be given in a future publication (Straub, in press).

\section{The stress edge}

The Shakura-Sunyaev model assumes that there is no torque at the inner edge of the disk, which in this model coincides with ISCO. Slim disk model assumes that there is no torque at the horizon of the black hole. This makes no assumption about the torque at the disk inner edge, but calculations prove that the torque is small there.

The zero-torque at the horizon is consistent with the small torque at the inner edge of slim disks, as Fig. 11 shows. The figure presents the relative importance of the torque $\mathcal{T}$

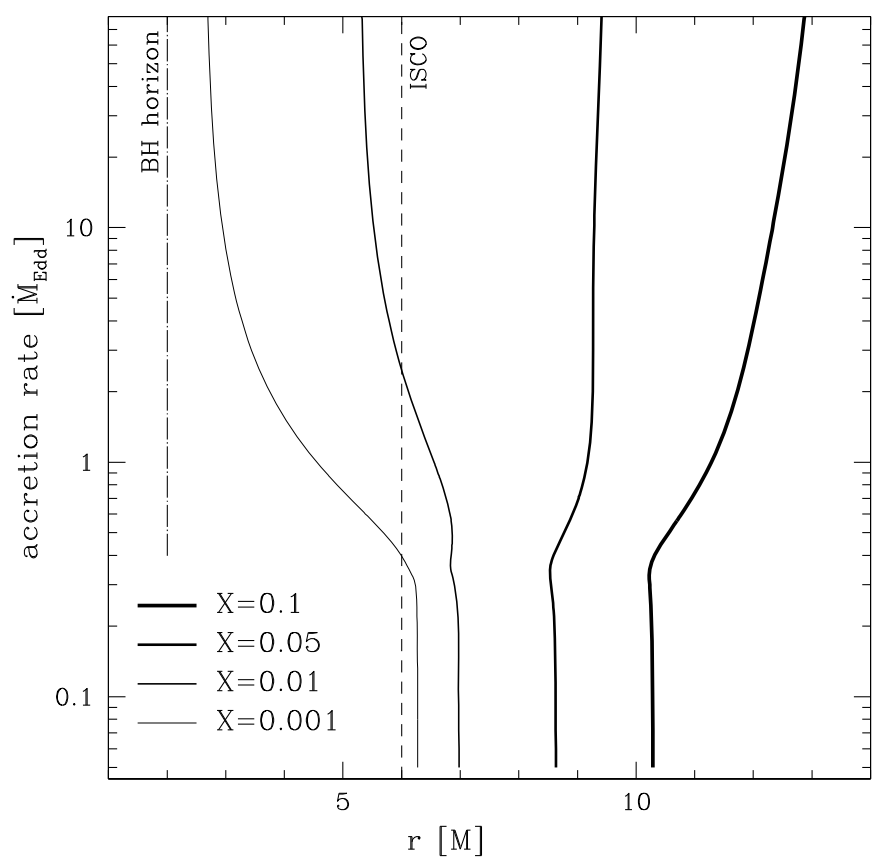

Fig. 12. Profiles of $r_{\text {str }}$ defined as the radius with given value of the torque parameter $\chi$ for $\alpha=0.01$. BH horizon and ISCO are also shown with dot-dashed and dashed lines, respectively.

by comparing it with the "advective" flux of angular momentum $\dot{M} j$ (cf. Eq. (B.1)). For the viscosity parameter $\alpha$ smaller than about 0.01 , the ratio $\chi=\mathcal{T} / \dot{M} j$ at both $r_{\text {pot }}$ and $r_{\text {son }}$ is smaller than 0.01 even for highly super-Eddington accretion rates, and for low accretion rates the ratio is vanishingly small, $\chi \approx 10^{-5}$. For high viscosity, $\alpha=0.5$, the ratio is very small for small accretion rates, $\chi<10^{-3}$ and smaller than about 0.1 for super-Eddington accretion rates (calculated at the sonic radius, as the disk enters the Bondi-like regime at these high accretion rates).

To define the stress inner edge $r_{\text {str }}$, one has to specify the characteristic value of the torque parameter $\chi$. Profiles of $r_{\mathrm{str}}$ for a few values of $\chi$ and $\alpha=0.01$ are shown in Fig. 12. The stress edge for $\chi \rightarrow 0$ is located at ISCO for low accretion rates. When the accretion rate exceeds $\sim 0.3 \dot{M}_{\text {Edd }}$, the edge departs from ISCO and moves closer to $\mathrm{BH}$ approaching its horizon with increasing $\dot{m}$. The behaviour of $r_{\text {str }}$ profiles for higher $(\gtrsim 0.1)$ values of $\chi$ is different - they move away from the $\mathrm{BH}$ as the angular momentum profiles become flatter with increasing accretion rates (compare Fig. 4).

In the case of disk-like accretion of a very low viscosity $\alpha \leq$ 0.01 , we find to high accuracy that

$r_{\text {pot }} \approx r_{\text {son. }}$

In this case, the "inner edge" inherits both the sonic edge and the potential spout edge properties, indicative of a small torque, which is indeed probably the case. By pushing the MHD numerical simulations to their limits, Shafee et al. (2008) and Penna et al. (2010) calculated a thin, $H / r \lesssim 0.1$, disk-like accretion flow, and demonstrated that its inner edge torque is small.

\section{The radiation edge}

As discussed in the previous section, the torque at $r_{\text {pot }}<r_{\text {ISCO }}$ is small, but non-zero and therefore there is also orbital energy dissipation at radii smaller than ISCO. Thus, some radiation does 
A\&A 521, A15 (2010)

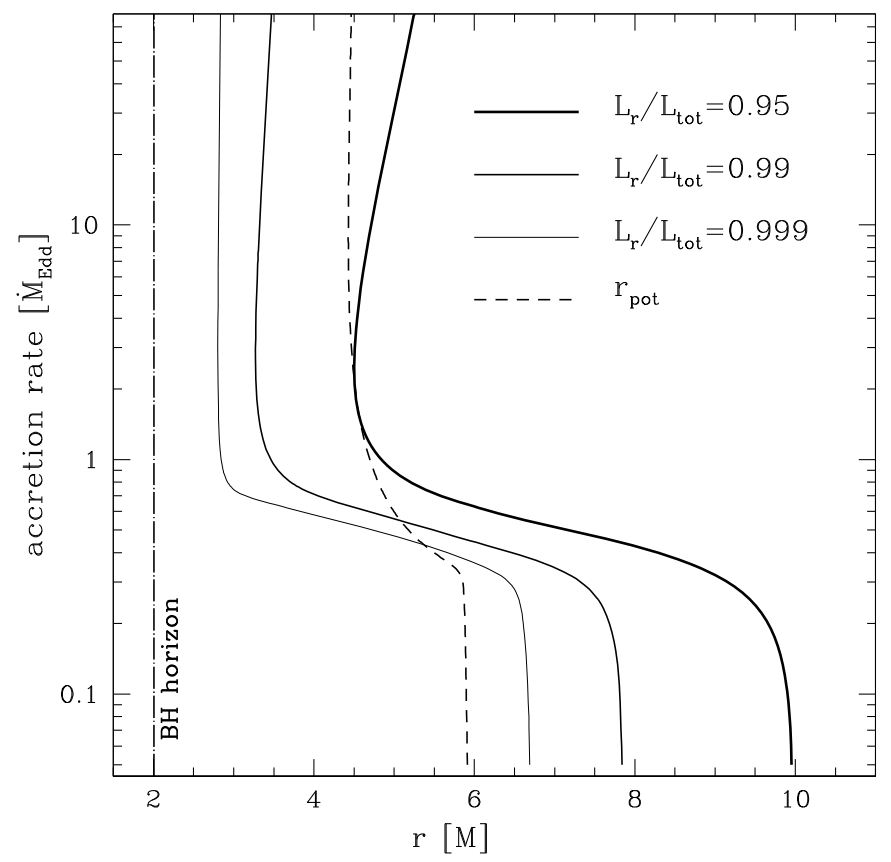

Fig. 13. "Luminosity edges" defining the inner radii of the area emitting a given amount of the total disk radiation. The lines are drawn for $95 \%$, 99\%, and $99.9 \%$ of the total emission. The dashed line shows the location of the potential spout inner edge $r_{\text {pot }}$. The gravitational suppression of the radiation has been taken into account.

originate in this region and the inner edge is not expected to coincide with the radiation edge, $r_{\text {rad }}<r_{\text {pot }}$. In Fig. 13, we present profiles of $r_{\text {rad }}$ defined as the radii limiting area emitting a given fraction of the disk total luminosity. For low accretion rates $\left(<0.3 \dot{M}_{\text {Edd }}\right)$, the disk emission terminates close to ISCO as the classical models of accretion disks predict. The locations of the presented $r_{\text {rad }}$ are determined by the regular Novikov \& Thorne flux radial profile. For higher accretion rates, the disk becomes advective and the maximum of the emission moves significantly inward. As a consequence of the increasing rate of advection (and resulting inward shift of $r_{\text {pot }}$ ), the efficiency of accretion drops down.

We emphasize that the location of the radiation edge is not determined by the location of the stress edge (as some authors seem to believe), but by the significant advection flux bringing energy into the region well below ISCO.

We define $r_{\text {out }} \gg r_{\mathrm{G}}$ to be the outer radius of the disk. The total luminosity of the disk can be estimated from

$L=\dot{M} e_{\mathrm{rad}}+(\mathcal{T} \Omega)_{\mathrm{rad}}-\dot{M} e_{\mathrm{out}}-(\mathcal{T} \Omega)_{\mathrm{out}}$,

$0=\dot{M} \mathcal{L}_{\text {rad }}+\mathcal{T}_{\text {rad }}-\dot{M} \mathcal{L}_{\text {out }}-\mathcal{T}_{\text {out }}$,

where $\mathcal{T}$ denotes the torque at a given radius. For both $\Omega_{\text {out }} \approx 0$, $e_{\text {out }} \approx 0$ one derives

$L=\dot{M}\left[e_{\mathrm{rad}}+\chi(\mathcal{L} \Omega)_{\mathrm{rad}}\right] \equiv \eta \dot{M}$,

where $\chi$ is the ratio of the viscous torque to the advective flux of angular momentum (see Figs. 11 and 12).

Because $\chi \ll 1$, the efficiency of accretion $\eta$ depends mainly on the specific energy at the inner edge, $e_{\text {rad }}$. The farther away the inner edge from ISCO (and closer to the black hole), the smaller the efficiency.

\section{The reflection edge}

The iron $\mathrm{K}_{\alpha}$ fluorescent line is an observed characteristic feature of many sources with black hole accretion disks (Miller 2006; Remillard \& McClintock 2006). The intensity and the shape of this line depends strongly on the physical conditions close to the inner edge. This has been discussed by many authors, including Reynolds \& Fabian (2008) who gave three conditions for line formation: (i) the flow has to be Thomson-thick in the vertical direction; (ii) disk has to be irradiated by an external source of $\mathrm{X}$-rays (hard X-ray irradiation plays a crucial role in the process of fluorescence and changes the ionization degree of matter); and (iii) the ionization state should be sufficiently low (iron cannot be fully ionized).

Nevertheless, since fluorescent iron-line emission depends on many aspects, such as the energy distribution of illuminating photons, temperature, ionization state, and density of the emitting matter as well as iron abundance, there is no obvious condition for the reflection edge (defined as the minimal radius where the reflection features originate). Additional computations of reflection models show that for some set of parameters iron fluorescent line may arise even from Thomson-thin matter (Dumont et al. 2002). In this paper, we assume that the reflection edge is connected to the condition

$\tau_{\mathrm{eff}}=\sqrt{\tau_{\mathrm{abs}}\left(\tau_{\mathrm{abs}}+\tau_{\mathrm{es}}\right)}>1$

However, one has to keep in mind that the effective optical depth at the iron line band may be much larger than the above, frequency-averaged value.

In Fig. 14, we show profiles of the effective optical depth $\tau_{\text {eff }}$ in different regimes of accretion rates for $\alpha=0.1$ and $a^{*}=0$. Three characteristic types of their behaviour are shown: sharp drop, maximum and monotonic at the top, middle, and bottom panels, respecively. The behaviour of different values of $\alpha$ and $a^{*}$ is qualitatively similar (but not quantitatively as in general $\tau_{\text {eff }}$ increases with decreasing $\alpha$ ). The top panel of the figure, corresponding to the lowest accretion rates, shows a sharp drop in $\tau_{\text {eff }}$ near ISCO. The same behavior was noticed previously e.g. by Reynolds \& Fabian (2008). The drop may clearly define the inner reflection edge $r_{\text {ref }} \approx r_{\text {ISCO }}$, limiting the radii where formation of the fluorescent iron line is prominent. The middle panel, corresponding to moderate accretion rates, shows a maximum in $\tau_{\text {eff }}$ near ISCO. The non-monotonic behaviour is caused by the regions of moderate radii outside ISCO being both radiation pressure and scattering dominated. We note, that the top of the maximum of $\tau_{\text {eff }}$ remains close to ISCO for a range of accretion rates, but for accretion rates higher than $0.3 \dot{M}_{\text {Edd }}$, it moves closer to the black hole with increasing $\dot{m}$ as the disk emission profile changes due to advection. The bottommost panel corresponds to super-Eddington accretion rates. The profiles are monotonic in $\tau_{\text {eff }}$ and define no characteristic inner reflection edge. Close to the black hole, these flows are effectively optically thin reaching $\tau_{\text {eff }}=1$ at about a few tens of gravitational radii.

When the effective optical depth of the flow becomes less then unity, our approximation of radiative transfer by diffusion with grey opacities (Eq. (A.8)) becomes invalid. In these cases, full radiative transfer through accretion disk atmospheres needs to be considered (e.g. Davis et al. 2005; Różańska \& Madej 2008). Nevertheless, our results allow us to estimate roughly how far from the black hole the iron line formation is most prominent, assuming that the disk is uniformly illuminated by an exterior X-ray source. For accretion rates lower than $0.3 \dot{M}_{\text {Edd }}$, the reflection edge is located very close to ISCO and we may identify the shape of the iron line with the gravitational and 

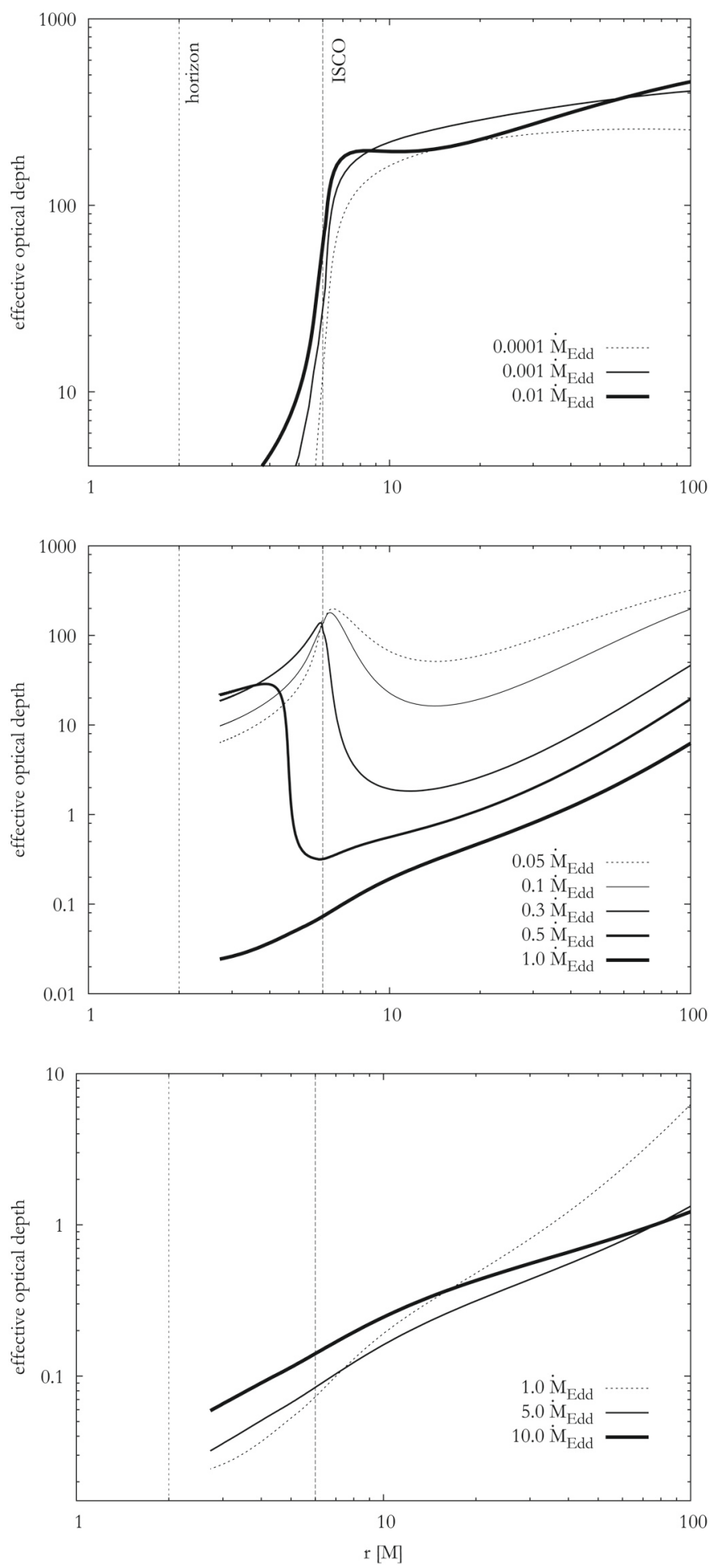

Fig. 14. Profiles of the effective optical depth $\tau_{\text {eff }}$ for $\alpha=0.1$ and $a=0$ in three different regimes of accretion rate. Vertical lines denote the locations of the BH horizon (dotted) and ISCO (dashed). Three types of behavior of $\tau_{\text {eff }}$ can be seen: sharp drop at ISCO at the lowest accretion rates, maximum near ISCO for moderate accretion rates, and monotonic everywhere for the highest accretion rates.

dynamical effects of the ISCO. In the case of higher but subEddington accretion rates, the maximum of the effective optical depth is located inside the ISCO, which may possibly allow us to study extreme gravitational effects on the iron line profile. However, the assumption that the line is formed at the ISCO is

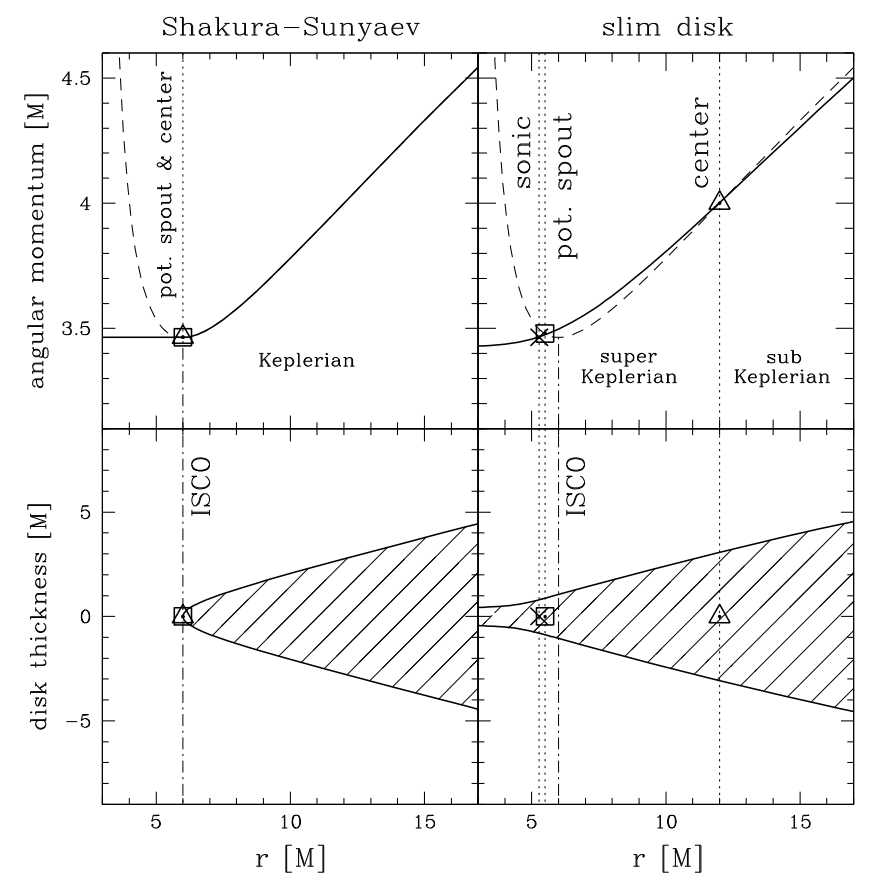

Fig. 15. The differences between Shakura-Sunyaev and slim disk picture of the disk inner edge (see text for a detailed explanation of the figure).

no longer satisfied. The super-Eddington flows have smooth and monotonic profiles of effective optical depth. Therefore, the reflection edge cannot be uniquely defined and no relation between the shape of the fluorescent lines and ISCO exists. Finally, we note that these lines may be successfully modeled by clumpy absorbing material and have nothing to do with relativistic effects (see e.g. Miller et al. 2009, and references therein). The role of the ISCO in determining the shape of the Fe lines was also questioned in the past (based on different reasoning) by Reynolds \& Begelman (1997), whose arguments were then refuted by Young et al. (1998).

\section{Conclusions}

We have addressed the inner edge issue by discussing the behavior of six differently defined "inner edges" of slim accretion disks around a Kerr black hole. We have found that the slim disk inner edges behave very differently than the corresponding Shakura-Sunyaev and Novikov-Thorne ones. The differences are qualitative and become important for the same range of luminosities independently of the BH spin. Even at moderate luminosities, $L / L_{\text {Edd }} \gtrsim 0.3$, there is no unique inner edge. Differently defined edges are located at different places. For nearly Eddington luminosities, the differences are huge and the notion of the inner edge loses all practical significance. We summarize the properties and locations of the six inner edges in Table 1.

We now conclude by presenting in Fig. 15 the differences between the Shakura-Sunyaev and slim-disk (in the disk-like case) treatment of the inner disk physics. The innermost part of a Shakura-Sunyaev disk is shown in the left column in Fig. 15, and the innermost part of a slim disk is shown in the right column. The upper panel compares the angular momentum in the disk (the solid line) with the Keplerian distribution (the dashed line). The ISCO, indicated by the dash-dotted line is at the radius 
Table 1. Summary of the results (specific numbers refer to the case $a^{*}=0$ ).

\begin{tabular}{|c|c|c|c|c|c|c|}
\hline \multirow[b]{2}{*}{$L / L_{\text {Edd }} \lesssim 0.3$} & $r_{\text {pot }}$ & $r_{\text {son }}$ & $r_{\mathrm{var}}$ & $r_{\mathrm{str}}$ & $r_{\text {rad }}$ & $r_{\text {ref }}$ \\
\hline & \multicolumn{6}{|c|}{$r_{\mathrm{in}} \approx r_{\mathrm{pot}} \approx r_{\mathrm{son}} \approx r_{\mathrm{var}} \approx r_{\mathrm{str}} \approx r_{\mathrm{rad}} \approx r_{\mathrm{ref}} \approx r_{\mathrm{ISCO}}$} \\
\hline$L / L_{\mathrm{Edd}} \gtrsim 0.3$ & $\begin{array}{c}\text { for } \alpha \lesssim 0.1 \text { moves } \\
\text { inward with increasing } \\
\dot{m} \text { down to } \sim r_{\mathrm{mb}} \text {; } \\
\text { for } \alpha \gtrsim 0.1 \text { and } \\
\text { sufficiently high } \dot{m} \text { disk } \\
\text { enters the Bondi } \\
\text { regime }- \text { undefined }\end{array}$ & $\begin{array}{l}\text { departs from } \\
\text { ISCO; } \\
\text { for } \alpha \ll 0.1 \\
r_{\mathrm{son}} \approx r_{\mathrm{mb}} ; \\
\text { for } \alpha \gtrsim 0.2 \\
r_{\mathrm{son}}>r_{\mathrm{ISCO}}\end{array}$ & undefined & $\begin{array}{c}\text { moves inward } \\
\text { with increasing } \\
L \text { down to } \mathrm{BH} \\
\text { horizon. }\end{array}$ & $\begin{array}{l}\text { moves inward } \\
\text { with increasing } \\
L \text { down to } \mathrm{BH} \\
\text { horizon. }\end{array}$ & $\begin{array}{c}\text { for } \\
0.3 \lesssim L / L_{\mathrm{Edd}} \lesssim 1.0 \\
r_{\mathrm{ref}}<r_{\mathrm{ISCO}} \\
\text { for } L / L_{\mathrm{Edd}} \gtrsim 1.0 \\
\text { undefined }\end{array}$ \\
\hline
\end{tabular}

where the Keplerian angular momentum has its minimum. The potential spout (a square) and the center (a triangle) are defined as the crossings of the angular momentum in the disk line with the Keplerian line. For slim disks, they occur at two different radii, on both sides of the ISCO. For Shakura-Sunyaev disks, they merge into one singular location at ISCO. The lower panel shows the cross-section of the disk. The slim disk everywhere has a finite thickness, while the Shakura-Sunyaen disk is singular at ISCO (it has a zero thickness there). The sonic radius (a cross) is where the accretion component of the velocity equals the local sound speed. In slim disks, the sonic point corresponds to a critical point of the set of differential equations, that by means of the regularity conditions defines the global eigensolution of the problem. The Shakura-Sunyaev disk is described by local algebraic equations and this global eigenvalue aspect is missing, thus the location of a sonic point is of no relevance.

Acknowledgements. This work was supported by Polish Ministry of Science grants N203 0093/1466, N203 304035, N203 380336, N203 00832/0709. A.S. acknowledges support from the Department of Astronomy at Kyoto University. M.A.A. acknowledges a professorship at Université Pierre et Marie Curie that supported his visit to Institut d'Astrophysique in Paris during which a part of research reported here was done. M.A.A. also acknowledges the Czech government grant MSM 4781305903. J.P.L. acknowledges support from the French Space Agency CNES.

\section{Appendix A: The Kerr geometry slim disks}

The Shakura-Sunyaev models are local: they are described by algebraic equations, valid at any particular (radial) location in the disk, independently of physical conditions at different locations. In contrast to this, the slim disk models of accretion disks are non-local. They are described by differential equations globally connecting physical conditions at all radial locations from infinity to the black hole horizon.

Models of slim disks were initially constructed by Abramowicz et al. (1988), who used the pseudo-Newtonian potential of Paczyński \& Wiita (1980) and Newtonian equations derived by Paczyński \& Bisnovatyi-Kogan (1981) and later improved by Muchotrzeb \& Paczyński (1982), Matsumoto et al. (1984), and Muchotrzeb (1983). The general relativistic version (the Kerr metric) of the slim disk equations was derived and elaborated on by Lasota (1994), Abramowicz et al. (1996), and Gammie \& Popham (1998), and later by Sacdowski (2009), who made several corrections and improvements to the results of the previous authors, and numerically constructed slim disk solutions for a wide range of parameters applicable to the X-ray binaries. In particular, he calculated solutions for the whole relevant range of accretion rates, from very sub-Eddingtonian, to moderately super-Eddingtonian ones. In this paper, we follow the notation and conventions adopted by Sadowski (2009). The Kerr geometry slim-disk equations adopted here are:

(i) The mass conservation

$\dot{M}=-2 \pi \Sigma \Delta^{1 / 2} \frac{V}{\sqrt{1-V^{2}}}$

where $\Sigma=\int_{-h}^{+h} \rho \mathrm{d} z \approx 2 H \rho$ is disk surface density and $V$ is the gas radial velocity as measured by an observer at fixed $r$, who co-rotates with the fluid. Here

$\Delta=r^{2}-2 M r-a^{2}$.

(For the Kerr metric description, see e.g. Kato et al. 2008, or any textbook on general relativity). Equation (A.1) has the same form in the Shakura-Sunyaev model.

(ii) The radial momentum conservation:

$\frac{V}{1-V^{2}} \frac{\mathrm{d} V}{\mathrm{~d} r}=\frac{\mathcal{A}}{r}-\frac{1}{\Sigma} \frac{\mathrm{d} P}{\mathrm{~d} r}$

where

$\mathcal{A}=-\frac{M A}{r^{3} \Delta \Omega_{k}^{+} \Omega_{k}^{-}} \frac{\left(\Omega-\Omega_{k}^{+}\right)\left(\Omega-\Omega_{k}^{-}\right)}{1-\tilde{\Omega}^{2} \tilde{R}^{2}}$,

$A=r^{4}+r^{2} a^{2}+2 M r a^{2}$

$\Omega=u^{\phi} / u^{t}$ is the angular velocity with respect to the stationary observer, $\tilde{\Omega}=\Omega-\omega$ is the angular velocity with respect to the inertial observer, $\Omega_{k}^{ \pm}= \pm M^{1 / 2} /\left(r^{3 / 2} \pm a M^{1 / 2}\right)$ are the angular frequencies of the co-rotating and counter-rotating Keplerian orbit and $\tilde{R}=A /\left(r^{2} \Delta^{1 / 2}\right)$ is the radius of gyration. In the ShakuraSunyaev model, this equation is a trivial identity $0=0$ because the radial pressure and velocity gradients vanish, and rotation is Keplerian, $\Omega=\Omega_{k}^{+}$.

(iii) The angular momentum conservation:

$\frac{\dot{M}}{2 \pi}\left(\mathcal{L}-\mathcal{L}_{\text {in }}\right)=\frac{A^{1 / 2} \Delta^{1 / 2} \gamma}{r} \alpha P$,

where $\mathcal{L}=-u_{\phi}$ is the specific angular momentum, $\gamma$ is the Lorentz factor, and $P=2 \mathrm{Hp}$ can be considered to be vertically integrated pressure. The constant $\alpha$ is the standard alpha viscosity parameter introduced by Shakura \& Sunyaev (1973). The constant $\mathcal{L}_{\text {in }}$ is the angular momentum at the horizon, unknown a priori. It provides an eigenvalue linked to the unique eigensolution to the set of slim disk differential equations constrained by proper boundary and regularity conditions. The algebraic 
Eq. (A.6) is the same as in the Shakura-Sunyaev model, except that the Shakura-Sunyaev model assumes that $\mathcal{L}_{\text {in }}=\mathcal{L}_{k}$ (ISCO).

(iv) The vertical equilibrium

$\frac{P}{\Sigma H^{2}}=\frac{\mathcal{L}^{2}-a^{2}\left(\epsilon^{2}-1\right)}{2 r^{4}}$,

where $\epsilon=u_{\mathrm{t}}$ is the conserved energy associated with the time symmetry. The same equation is valid for the Shakura-Sunyaev model.

(v) The energy conservation

$-\frac{\alpha P A \gamma^{2}}{r^{3}} \frac{\mathrm{d} \Omega}{\mathrm{d} r}-\frac{32}{3} \frac{\sigma T^{4}}{\kappa \Sigma}=-\frac{\dot{M}}{2 \pi r \rho} \frac{1}{\Gamma_{3}-1}\left(\frac{\mathrm{d} p}{\mathrm{~d} r}-\Gamma_{1} \frac{p}{\rho} \frac{\mathrm{d} \rho}{\mathrm{d} r}\right)$

where $T$ is the disk central temperature. The right-hand side of this equation represents the advective cooling and vanishes in the Shakura-Sunyaev model. Because rotation is Keplerian in the Shakura-Sunyaev model, $\Omega=\Omega_{k}^{+}$, which means that $\Omega$ is a known function of $r$ and therefore that the first term on the lefthand side (which represents viscous heating) is algebraic. The second term, which represents the radiative cooling (in a diffusive approximation) is similar in the Shakura-Sunyaev model.

\section{Appendix B: No torque at the black hole horizon}

The assumption of a (vanishingly) small torque in the region between black hole and accretion disk is physically well motivated. We recall that the very meaning of a torque $\mathcal{T}$ is that it transports angular momentum without transporting mass. Correspondingly, the total angular momentum flux $\dot{J}$ through a surface equals, in general,

$\dot{J}=\dot{M} j+\mathcal{T}$,

where $\dot{M}$ is the mass flux and $j$ is the angular momentum density (per unit mass). However, the torque is only a phenomenological concept. Microscopically, the flux $\dot{J}$ should be seen as a difference of material fluxes that come from the opposite sides of the surface, $\dot{J}=\left[\dot{M}_{+} j_{+}\right]-\left[\dot{M}_{-} j_{-}\right]$. One also has $\dot{M}=\dot{M}_{+}-\dot{M}_{-}$, and $j=\left(j_{+} \dot{M}_{+}+j_{-} \dot{M}_{-}\right) /\left(\dot{M}_{+}+\dot{M}_{-}\right)$. Microscopically then, the torque is equal to $\mathcal{T}=2 \dot{M}_{+} \dot{M}_{-}\left(j_{+}-j_{-}\right) /\left(\dot{M}_{+}+\dot{M}_{-}\right)$. It vanishes necessarily when all matter crosses the surface in only one direction, i.e. when either $\dot{M}_{+}=0$ or $\dot{M}_{-}=0$. As the one-side matter flux is the only fundamental property of the black horizon, one concludes that there should be no torque at the black hole surface.

Since the Blandford \& Znajek (1977) process energizes the jet (and disk) by extracting the rotational energy of a black hole by means of electromagnetic braking, some astrophysicists argue that in this case there must be a "Maxwell" torque between the black hole and outside matter. However, by looking at the Blandford-Znajek process from the quantum electrodynamics perspective, one can see only ingoing, but no outgoing photons. Thus, there is only a one-way traffic of photons, and no torque possible. The photons with negative energy and angular momentum that are present in the ergosphere, are responsible for the slowing down of the hole, similarly to the negative energy particles in the classic Penrose process that must necessarily also have negative angular momentum. This point of view, that the Blandford-Znajek process is an electromagnetic version of the Penrose process, was discussed in the context of the classical Maxwell electrodynamics (in Kerr geometry) by several authors, in particular most forcefully by Komissarov (2008).

Here, we generalize Komissarov's point to any material field, not only the electromagnetic one. Following Komissarov, we consider the local ZAMO (or FIDO) observer in the Kerr geometry. His four velocity in terms of the Killing vectors $\eta^{i}$ (time symmetry) and $\xi^{i}$ (axial symmetry) is given by $n^{i}=q\left(\eta^{i}+\omega \xi^{i}\right)$, where $\omega$ is the angular velocity of frame dragging, and $q>0$ follows from normalization $n^{i} n^{k} g_{i k}=-1$. We now consider a general matter or field, described by an unspecified stress-energy tensor $T^{i}{ }_{k}$. The energy flux in the ZAMO frame is $E^{i}=-T^{i}{ }_{k} n^{k}$. The rate at which energy is acquitted by the black hole is

$E=-\int T^{i}{ }_{k} n^{k} \mathrm{~d} N_{i}>0$,

where $\int \mathrm{d} N_{i}$ is the surface integral over the horizon. The inequality sign follows from the locally measured energy having to be positive. The above integral may by transformed into

$0<E=-\int q T_{k}^{i}\left(\eta^{k}+\omega \xi^{k}\right) \mathrm{d} N_{i}=q_{H}\left(E_{\infty}-\omega_{H} J_{\infty}\right)$,

where the index $H$ denotes horizon, and $E_{\infty}$ and $J_{\infty}$ are the "energy at infinity" and the "angular momentum at infinity" acquired by the black hole absorbing the corresponding fluxes of these quantities defined by,

$E_{\infty}^{i}=-T_{k}^{i} \eta^{k}, \quad J_{\infty}^{i}=T_{k}^{i} \xi^{k}$

From Eq. (B.3), one concludes that $E_{\infty}>J_{\infty} \omega_{H}$. As in the classic Penrose process, the necessary condition for the extraction of energy at infinity is that the energy (at infinity) absorbed by a black hole is negative, $E_{\infty}<0$. This is equivalent to $J_{\infty} \omega_{H}<0$. Thus, in a way fully analogous to the line of arguments that is made when discussing the Penrose process, one may say that if energy at infinity increases because the black hole absorbed a negative-at-infinity energy, then the black hole must also slow down by absorbing matter or electromagnetic flux of negative angular momentum.

\section{References}

Abramowicz, M. A. 1981, Nature, 294, 235

Abramowicz, M. A. 1985, PASJ, 37, 727

Abramowicz, M. A., \& Zurek, W. H. 1981, ApJ, 246, 314

Abramowicz, M. A., Jaroszyński, M., \& Sikora, M. 1978, A\&A, 63, 221

Abramowicz, M. A., Czerny, B., Lasota, J.-P., \& Szuszkiewicz, E. 1988, ApJ, 332,646

Abramowicz, M. A., Bao, G., Lanza, A., \& Zhang, X.-H. 1991, A\&A, 245, 454 Abramowicz, M. A., Lanza, A., Spiegel, E. A., \& Szuszkiewicz, E. 1992, Nature, 356,41

Abramowicz, M. A., Chen, X.-M., Granath, M., \& Lasota, J.-P. 1996, ApJ, 471, 762

Afshordi, N., \& Paczyński, B. 2003, ApJ, 592, 354

Agol, E., \& Krolik, J. H. 2000, ApJ, 528, 161

Balbus, S. A., \& Hawley, J. F. 1991, ApJ, 376, 214

Barret, D., Kluźniak, W., Olive, J. F., Paltani, S., \& Skinner, G. K. 2005, MNRAS, 357, 1288

Bath, G. T., Evans, W. D., \& Papaloizou, J. 1974, MNRAS, 167, 7p Beckwith, K., Hawley, J. F., \& Krolik, J. H. 2008, MNRAS, 390, 21 Blandford, R. D., \& Znajek, R. L. 1977, MNRAS, 179, 433

Bondi, H. 1952, MNRAS, 112, 195

Boutelier, M., Barret, D., Lin, Y., \& Török, G. 2010, MNRAS, 401, 1290

Davis, S. W., Blaes, O. M., Hubeny, I., \& Turner, N. J. 2005, ApJ, 621, 372

Dumont, A.-M., Czerny, B., Collin, S., \& Życki, P. T. 2002, A\&A, 387, 63

Gourgoulhon, E., \& Jaramillo, J. L. 2006, Phys. Rep., 423, 159

Hawley, J. F., Balbus, S. A., \& Stone, J. M. 2001, ApJ, 554, 49

Esin, A. A., McClintock, J. E., \& Narayan, R. 1997, ApJ, 489, 865

Frank, J., King, A., \& Raine, D. 2002, Accretion Power in Astrophysics, 3rd edition (Cambridge University Press)

Gammie, C. F. 1999, ApJ, 522, L57

Gammie, C. F., \& Popham, R. 1998, ApJ, 498, 313

Igumenshchev, I. V., \& Beloborodov, A. M. 1997, MNRAS, 284, 767 
Jaroszyński, M., Abramowicz, M. A., \& Paczyński, B. 1980, Acta Astr., 30, 1 Kato, S., Fukue, J., \& Mineshige, S. 2008, Black-Hole Accretion Disks Towards a New Paradigm, 2nd edition (Kyoto University Press)

Kato, S. 2001, PASJ, 53, 1

Krolik, J. H. 1999 ApJ, 515, L73

Krolik, J. H., \& Hawley, J. F. 2002, ApJ, 573, 754

Komissarov, S. S. 2006, MNRAS, 368, 993

Komissarov, S. S. 2008, J. Korean Phys. Soc., 54, 2503 [arXiv: 0804 . 1912]

Kozłowski, M., Abramowicz, M. A., \& Jaroszyński, M. 1978, A\&A, 63, 209

Lasota, J. P. 1994, in Theory of Accretion Disks - 2, ed. W. J. Duschl, J. Frank, F. Meyer, E. Meyer-Hofmeister, \& W. M. Tscharnuter, NATO ASIC Proc., 417,341

Matsumoto, R., Kato, S., Fukue, J., \& Okazaki, A. T. 1984, PASJ, 36, 71

McClintock, J. E., Shafee, R., Narayan, R., et al. 2006, ApJ, 652, 518

Middleton, M., Done, C., Ward, M., Gierliśki, M., \& Schurch, N. 2009, MNRAS, 394,250

Miller, J. M. 2006, Astron. Nachr., 327, 997

Miller, L., Turner, T. J., \& Reeves, J. N. 2009, MNRAS, 399, L69

Muchotrzeb, B., \& Paczyński, B. 1982, Acta Astr., 32, 1

Muchotrzeb, B. 1983, Acta Astr., 33, 79

Muchotrzeb-Czerny, B. 1986, Acta Astr., 36, 1
Narayan, R., \& Yi, I. 1988, ApJ, 444, 231

Narayan, R., Igumenshchev, I. V., \& Abramowicz, M. A. 2003, PASJ, 55. L69

Noble, S. C., Krolik, J. H., \& Hawley, J. F. 2010, ApJ, 711, 959

Novikov, I. D., \& Thorne, K. S. 1973, Black Holes, Les Astres Occlus, 343

Paczyński, B. 1998, unpublished [arXiv: astro-ph/9812047]

Paczyński, B. 2000, unpublished [arXiv: astro-ph/0004129]

Paczyński, B., \& Wiita, P. J. 1980, å, 88, 23

Paczyński, B., \& Bisnovatyi-Kogan, G. 1981, Acta Astr., 31, 283

Pecháček, T., Karas, V., \& Czerny, B. 2008, A\&A, 487, 815

Penna, R. F., McKinney, J. C., Narayan, R., et al. 2010, MNRAS, 408, 752

Pringle, J. E. 1981, Ann. Rev. Ast. Ap., 19, 137

Remillard, R. A., \& McClintock, J. E. 2006, ARA\&A, 44, 49

Reynolds, C. S., \& Begelman, M. C. 1997, ApJ, 488, 109

Reynolds, C. S., \& Fabian, C. 2008, ApJ, 675, 1048

Różańska, A., \& Madej, J. 2008, MNRAS, 386, 1872

Sạdowski, A. 2009, ApJS, 183, 171

Schnittman, J. D. 2005, ApJ, 621, 940

Shafee, R., McKinney, J. C., Narayan, R., et al. 2008, ApJ, 687, L25

Shakura, N. I., \& Sunyaev, R. A. 1973, A\&A, 24, 337

Wagoner, R. V. 1999, Phys. Rep., 311, 259

Young, A. J., Ross, R. R., \& Fabian, A. C. 1998, MNRAS, 300, L11 